\title{
Contents, Vol. 91, 1937
}

\section{Inhaltsverzeichnis.}

Eigenarbeiten.

Seite

Adda, Viktor, Über einen Fall von metastatischem Carcin-

om im Ciliarkörper 329

Bergiiieister, K., Über Keratitis parenchymatosa bei Lues

congenita der zweiten Generation 264

Blobner, Ferdinand, Z, u $\tau$ rezidivierenden Hypopyoniritis 129

Bokermann, Ludwig, Heilung eines Netzhautglioms durch

Röntgenstrahlen $\quad 348$

Bucklers, Max, Katarakta filiformis 338

Csillag, F., Dynamische Faktoren bei der intrakapsulären

Starextraktion 158

-, Vorschläge zu einer neuen Operationstechnik zur Zer-reißung der Zonulafasern und zum

Entfernen derLinse in der Kapsel durch Stürzen 171

David, H'., siehe Pillat, A.

Gerke, /., Die künstliche Wiederherstellung des Ductus

nasolacrimalis

50

Graf, Kornelja, Chronisches intermittierendes Hornhaut-

ödem als Ausdruck einer Angioneurose 278

Пaldimann, Carl, Über Keratitis parenchymatosa bei Lues

congenita der zweiten Generation 183

Hamburger, F. A., Prophylaxe gegen Ulcus serpens mit

Collargolsalbe 265

Kale $\uparrow f$, Eine vereinfachte Modifikation der Dacryocysto-

rhinostomia externa 140

Kreibig, Wilhelm, Eine Blepharospasmusbrille .... 289

Maiiksch, Id., Pollenallergie als Ursache einer Keratitis

superficialis 343

Ohm, /., Der Nystagmus bei der totalen angeborenenFarbenblindheit und seine Beziehungen zum Augen-zittern der Bergleute nebst Bemerkungen zur Erklärungder Farbenblindheit

Pillat, A., und H. David, Über Tularämie beim Menschen

in Österreich 1

Riedl, Franz, Gumma der Bulbusbindehaut bei Lues congenita

Verschiedenes.

Kraupa, E., L. Böhms Vererbungsgesetze der Kurzsichtig-

keit 183

IV Inhaltsverzeichnis.

Seite Kraitpa, E., Krebs, durch fehlerhaften Brillensteg ver- 
ursacht 185

Curin, Juraj, Die juvenile Epitheldystrophie 185

Berichte liber die ophthalmologische Literatur.

Aderhaut $>$ Netzhaut (Berichtsjahr vom 1. Januar bis 31. De-

zember 1935). Von Prof. W. Riehm, Gießen ... 53

Entwicklungsgeschichte des Auges, Mißbildungen, Anthro-pologie (Berichtszeitraum: vom 1.

Juli 1935 bis 30. Juni1936). Von R. Seefelder, Innsbruck 93

Geschichte der Augenheilkunde. Von R. See/elder, Innsbruck 108

Normale und pathologische Anatomie (Berichtsjahr 1935).

Von Doz. Dr. Rudolf Schneider, Graz 112

Physiologische Optik (Berichtsjahr vom 1. Okt. 1935 bis

30. Sept. 1936). Von Dr. Rud. Stieber, Innsbruck . 187

Psychologische Optik (Berichtsjahr vom 1. Okt. 1935 bis

30. Sept. 1936). Von Dr. Rud. Stieber Innsbruck . . 208

Vererbung und Auge (Berichtsjahr 1935/36).

Von A. Franceschetti, Genf 292

Ophthalmologische Therapie I. Medikamentöse Therapie.

Von Dr. Eberhard Kunz, Berlin-Charlottenburg . . . 352

Ophthalmologische Therapie II. Operative Therapie. Von

Prof. Wolfgang Hoffmann, Königsberg i. Pr. . . . 360

Ophthalmologische Therapie III. Physikalische und

Strahlentherapie. Von Prof. Wolfgang Hoffmann, Königsberg i. Pr 369

Gesellschaftsberichte.

Ver < einiguing mittelcleutsicher Augenärztie:

Taguing am 30. November und 1. Dezember 1935 . . . 233

Taguing am 19. und 20. September $1936 \quad 248$

Ophthalmologische Gesellschaft in Wien:

Sitzung vom 19. Oktober $1936 \quad$ 214Sitzung vom 7. Dezember 193632029.

Jahresversammlung der Schweizerischen Ophthalmologiscben Gesellschaft. Sitzung vom 13.

und 14. Juni 1936

Diagnose und Therapie $\quad 124,261,325,374$

Fortbildungskurs

127

Einladung 128

S. Sachregister zu Band $91 \quad 379$

Namenregister zu Band 91 